\title{
Caractérisations physicochimiques de biofilms synthétisés à partir de la pectine et de la gélatine
}

\author{
Gildas Komenan GBASSI ${ }^{1 *}$, Karima ZAHER ${ }^{2}$, Fernand Seri YOLOU ${ }^{1}$, Meriem \\ MERBAH $^{2}$ et Michèle AKE ${ }^{1}$. \\ ${ }^{I}$ Département de Chimie Analytique, Chimie Générale et Minérale, Université de Cocody, \\ UFR Sciences Pharmaceutiques, 01 BP V 34, Abidjan, Côte d'Ivoire. \\ ${ }^{2}$ Département de Génie des Procédés, Faculté des Sciences de l'Ingénieur, Université Ferhat \\ ABBAS, 19000 Sétif, Algérie. \\ *Auteur correspondant, E-mail : gildas.gbassi@laposte.net; Tél: + 22522472217
}

\section{RESUME}

Trois types de biofilms à base de polymères naturels ont été préparés à partir de la pectine, un polyélectrolyte anionique, et de la gélatine, une espèce cationique. Les propriétés physicochimiques (épaisseur, perméabilité à la vapeur d'eau et pouvoir gonflant) des biofilms ont été déterminées; de même, leur morphologie a été évaluée. Au niveau micrométrique, les épaisseurs des biofilms étaient respectivement de $101,2 \pm 0,2 \mu \mathrm{m} ; 64,6 \pm 0,1 \mu \mathrm{m}$ et $39,0 \pm 0,1 \mu \mathrm{m}$ pour la gélatine, le mélange gélatine/pectine et la pectine. Le pouvoir gonflant était de $300 \%$ pour la gélatine, $215 \%$ pour le mélange gélatine/pectine et $90 \%$ pour la pectine. La perméabilité à la vapeur d'eau était de $9,20 \mathrm{~g} \mathrm{~mm} / \mathrm{m}^{2} \mathrm{~h} \mathrm{KPa}$ pour la gélatine; $6,59 \mathrm{~g} \mathrm{~mm} / \mathrm{m}^{2} \mathrm{~h} \mathrm{KPa}$ pour le mélange gélatine/pectine et $3,98 \mathrm{~g} \mathrm{~mm} / \mathrm{m}^{2} \mathrm{~h} \mathrm{KPa}$ pour la pectine. Au niveau morphologique, des différences significatives ont été observées. Toutefois, les différents films formulés ont mis en évidence des potentialités physicochimiques intéressantes.

(c) 2009 International Formulae Group. All rights reserved.

Mots clés : Biofilms, polymères naturels, analyse physicochimique.

\section{INTRODUCTION}

L'usage des polymères naturels communément appelés biopolymères connaît un développement remarquable depuis ces dernières années en raison de leurs nombreuses applications notamment comme matériaux d'emballage (Avena-Bustillos et al., 1997) ou agents d'enrobage (Tapia et al., 2007). Leurs structures chimiques présentent des propriétés particulièrement intéressantes dont la plus utilisée est la biodégradabilité (Jo et al., 2005). Ce sont donc des matériaux aptes à subir un processus de dégradation sous l'action de phénomènes physicochimiques. L'amélioration des propriétés de ces polymères pour des applications nouvelles suscite un regain d'intérêt de la communauté scientifique notamment dans la mise au point de biofilms. Ainsi, des biofilms ont été synthétisés avec des polymères naturels comme la gélatine (Garcia et al., 2004), le chitosan (Dong et al., 2006; Dutta et al., 2009), l'alginate (Zactiti and Kieckbusch, 2006; Tapia et al., 2007). Aussi des biofilms issus de l'association de polymères naturels sont décrits dans la littérature comme les mélanges protéines du lactosérum/amidon (Zaleska et al., 2001), pectine/chitosan (Ghaffari et al., 2007) ou alginate/pectine (Da silva et al., 2009). On reconnaît à ces biofilms des propriétés d'autoadhésivité et de perméabilité. D'après Arwidsson (1991), la libération d'une substance active à partir d'un biofilm dépend fortement de la perméabilité 
de ce dernier. Par ailleurs Aulton (1982) a suggéré une évaluation rationnelle des caractéristiques d'un biofilm pour prédire son application ultérieure. Dans ce travail, nous nous sommes proposés d'étudier deux polymères naturels: la gélatine, un composé de nature protéique, et la pectine, un composé de nature polysaccharidique. La gélatine est obtenue par hydrolyse du collagène, ce dernier est le constituant majeur de la peau et des tissus osseux (Tabata and Ikada, 1998). La gélatine est largement utilisée dans l'industrie médicale comme pansement pour blessures, garniture absorbante ou adhésif (Neumann et al., 1981) et plus particulièrement comme matériel biopharmaceutique pour la libération contrôlée des médicaments (Fukunaka et al., 2002). La gélatine contient un total de 18 acides aminés en différentes concentrations, la liaison de ces acides forme des chaînes polypeptidiques, chaque chaîne contient environ mille acides aminés (Gomez-Guillen et al., 2002). La structure globale de la gélatine est une molécule en forme de baguette se composant des structures hélicoïdales primaire, secondaire et tertiaire (Kozlov and Burdygina, 1983; Ross-Murphy, 1992). Quant à la pectine, elle est également utilisée dans le domaine médical pour ses activités biologiques diverses (Samuelsen et al., 1996). La pectine est un polymère naturel de structure très complexe caractérisée par deux paramètres (degré d'acétylation et degré de méthylation) qui conditionnent l'ensemble de ses propriétés et mécanismes d'action (Dea and Madden, 1986; Lofgren and Hermansson, 2007; Lutz et al., 2009). L'objectif de cette étude était de formuler des biofilms à partir de ces deux polymères naturels et d'évaluer certaines de leurs propriétés physicochimiques telles que le pouvoir gonflant, la perméabilité à la vapeur d'eau et la morphologie.

\section{MATERIEL ET METHODES Matériel}

La pectine de type A (hautement méthylée) provient de la firme CP Kelco (Grossenbrode, Allemagne). La gélatine de type B (point isoionique compris entre 4,8 et $5,2)$ provient de la pharmacie Baille 181 (Marseille, France). Le glutaraldéhyde et l'azide de sodium ont été fournis par la firme Sigma-Aldrich (Steinheim, Allemagne).

\section{Préparation des biofilms}

La préparation des biofilms a été adaptée à partir de protocoles précédemment décrits dans la littérature (Dong et al., 2006; Ghaffari et al., 2007). Une solution aqueuse à $5 \%(\mathrm{~m} / \mathrm{v})$ de chaque polymère a été préparée à température ambiante sous une agitation constante durant 30 minutes. Un mélange de gélatine/pectine dans les proportions de 3:2 (v/v) a été réalisé à partir des précédentes solutions. Une pincée d'azide de sodium a été ajoutée à chacune des solutions pour prévenir une éventuelle contamination microbienne. Chaque solution a été placée dans un bain marie sous une agitation douce puis chauffée à $40{ }^{\circ} \mathrm{C}$ pour la gélatine, $70{ }^{\circ} \mathrm{C}$ pour le mélange et $80{ }^{\circ} \mathrm{C}$ pour la pectine pendant 30 minutes. Lorsque les solutions sont apparues limpides, $5 \mathrm{ml}$ de chacune d'elles ont été dispersés dans une boite de Pétri en polystyrène de $8,5 \mathrm{~cm}$ de diamètre. Le séchage s'est effectué à l'air libre à température ambiante durant 3 à 4 jours. Après cette période, $20 \mathrm{ml}$ de glutaraldéhyde à $0,02 \%(\mathrm{v} / \mathrm{v})$ ont été ajoutés aux biofilms secs pour initier la réaction de réticulation qui s'est poursuivie pendant $24 \mathrm{~h}$. Les biofilms ont ensuite été rincés deux fois à l'eau distillée puis séchés à l'étuve $\left(40{ }^{\circ} \mathrm{C}\right)$ pendant $2 \mathrm{~h}$.

\section{Caractérisation des biofilms Mesure de l'épaisseur des biofilms}

L'épaisseur des films a été mesurée à l'aide d'un micromètre numérique (Blet, Lyon, France) avec une précision de $0,1 \mu \mathrm{m}$. Les mesures ont été prises au hasard à 5 endroits différents du film.

\section{Détermination de la perméabilité à la vapeur d'eau}

La perméabilité est la quantité de gaz (ici vapeur d'eau) transmise à travers une surface (ici biopolymère) par unité de temps, de pression et/ou d'épaisseur. Elle permet de caractériser les propriétés d'étanchéité ou de fuite vis-à-vis d'un fluide. La coupe de perméabilité de Payne (Figure 1) d'un diamètre de $3,5 \mathrm{~cm}$ a été utilisée dans cette étude.

L'intérieur de la coupe a été rempli par $10 \mathrm{ml}$ d'eau distillée (humidité relative fixée à $100 \%$ ). Chaque biofilm a été découpé, pesé, puis placé entre l'anneau étanche et la coupe. Cet anneau a été positionné de sorte à exposer une surface exacte de biofilm de $10 \mathrm{~cm}^{2}$. Un 
échantillon témoin a servi de référence et a été fixé sur une seconde coupe ne contenant pas d'eau distillée. L'ensemble biofilm/coupe a été pesé de façon précise $( \pm 0,001 \mathrm{~g})$ puis conservé dans un dessiccateur contenant du pentoxide de phosphore $\left(\mathrm{P}_{2} \mathrm{O}_{5}\right)$ et des grains de silice (humidité relative fixée à $0 \%$ ). A intervalle de temps réguliers $(24 \mathrm{~h}, 48 \mathrm{~h}, 72 \mathrm{~h}$, $96 \mathrm{~h}$ et $120 \mathrm{~h}$ ), les biofilms ont été pesés de nouveau. Le profil de la perte de masse a été tracé en fonction du temps pour chaque biofilm. La perméabilité à la vapeur d'eau (PVE) a été calculée en utilisant la formule donnée par Akhgari et al. (2006).

$$
P V E=\frac{m}{t} \times \frac{E}{S \times P o \times(H R 1-H R 2)},
$$

où $m / t\left(\mathrm{~g} \mathrm{~h}^{-1}\right)$ est la modification de masse de la $120^{\mathrm{è}}$ heure, $E(\mathrm{~mm})$ l'épaisseur du biofilm, $S\left(\mathrm{~m}^{2}\right)$ la surface du biofilm exposé à la vapeur d'eau, $P o(\mathrm{KPa})$ la pression de vapeur de l'eau pure, HRl-HR2 est le gradient d'humidité relative. A $25^{\circ} \mathrm{C}, P o$ est égal à 3,159 KPa.

\section{Détermination du pouvoir gonflant}

Une surface de $2 \mathrm{~cm}^{2}$ de biofilm sec a été pesée de façon précise $( \pm 0,1 \mathrm{mg})$ puis immergée dans un bécher contenant $100 \mathrm{ml}$ d'eau distillée $(\mathrm{pH} 7)$ sous une agitation douce et constante, à température ambiante pendant $2 \mathrm{~h}$. A intervalle de temps réguliers $(30 \mathrm{~min}$, $60 \mathrm{~min}, 90 \mathrm{~min}$ et $120 \mathrm{~min}$ ), le film a été retiré du bécher, égoutté à l'aide d'un papier filtre puis pesé de nouveau. L'indice de gonflement ( $I g)$ a été calculé par la formule suivante :

$$
\operatorname{Ig}(\%)=\frac{M f g-M f s}{M f g} \times 100 ;
$$

où $M f g$ est la masse $(\mathrm{mg})$ du film gonflé et $M f s$ la masse (mg) du film sec.

\section{Evaluation de la morphologie des biofilms}

Les investigations morphologiques à la surface des biofilms ont été réalisées à l'aide de la microscopie électronique à balayage (MEB, Philips XL 20, Oregon, USA). Les échantillons ont été pulvérisés sur un métal hybride or/palladium dans une chambre sous vide avant l'analyse microscopique.

\section{Analyse statistique}

Le logiciel Excel $^{\circledR} \quad$ (Windows $\mathrm{XP}^{\circledR}$ version 2003) a été utilisé pour le traitement statistique des données brutes. Les valeurs moyennes et les écart-types ont été calculés.
Le test de Student a été appliqué pour comparer les moyennes des données. Toute valeur de $\mathrm{p}<0,05$ a été considérée comme statistiquement significative.

\section{RESULTATS}

Aspect macroscopique

L'image photographique des films de gélatine (A), pectine (B) et du mélange (C) est donnée à la figure 2. Sur le plan visuel, les différents films sont transparents. Au toucher, les films sont lisses et flexibles.

\section{Epaisseur des biofilms}

L'épaisseur moyenne du film de gélatine est de 101,2 $\pm 0,2 \mu \mathrm{m}$; celui du mélange $64,6 \pm 0,1 \mu \mathrm{m}$ et celui de la pectine $39,0 \pm 0,1 \mu \mathrm{m}$. Le film de gélatine est environ 2,6 fois plus épais que celui de la pectine et 1,57 fois plus épais que celui du mélange. Une différence significative d'épaisseur a été obtenue entre ces films ( $p>0,05)$.

\section{Perméabilité à la vapeur d'eau (PVE)}

La PVE des films de pectine, du mélange et de gélatine étaient respectivement de 3,98; 6,59 et 9,20 g mm/ $\mathrm{m}^{2} \mathrm{~h} \mathrm{kPa}$. La PVE de la pectine est 2,3 fois moins élevée que celle de la gélatine et 1,66 fois moins élevée que celle du mélange. Une différence significative de perméabilité a été obtenue entre ces films ( $p>0,05)$. Cette PVE se calcule à partir d'une formule mathématique en tenant compte de la perte de masse de la dernière heure des films exposés à la vapeur d'eau. D'après la figure 3 , la perte de masse est constante pour les trois films mais plus élevée avec la pectine. On observe une corrélation linéaire de la quantité de vapeur d'eau transmise par unité de temps.

\section{Pouvoir gonflant}

La figure 4 présente le profil de gonflement des films de gélatine, du mélange gélatine/pectine et de la pectine. La gélatine possède une capacité d'absorption de l'ordre de $300 \%$ à 30 min comparée à la pectine (95\%) et au mélange (215\%). Le pouvoir gonflant de la gélatine est 3,16 fois plus élevé que celui de la pectine et 1,40 fois plus élevé que celui du mélange. Une différence d'absorption significative a été obtenue entre ces films $(p>0,05)$. 


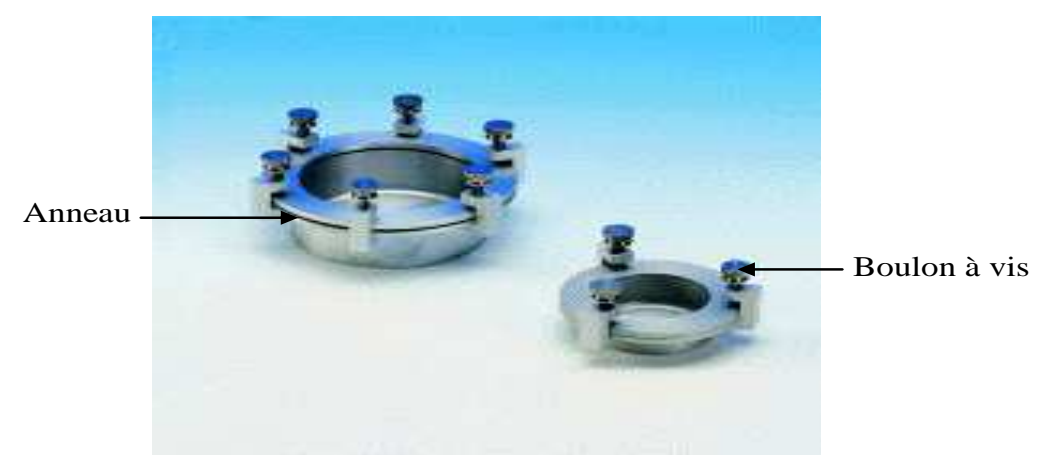

Figure 1: Coupe de perméabilité de Payne (modèle Elcometer 5100).

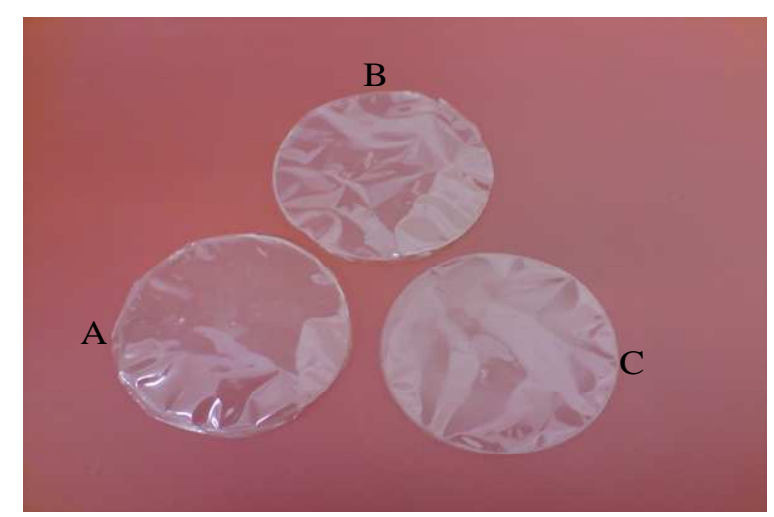

Figure 2: Image photographique des différents films (A: gélatine ; B : gélatine/pectine ; C: pectine).

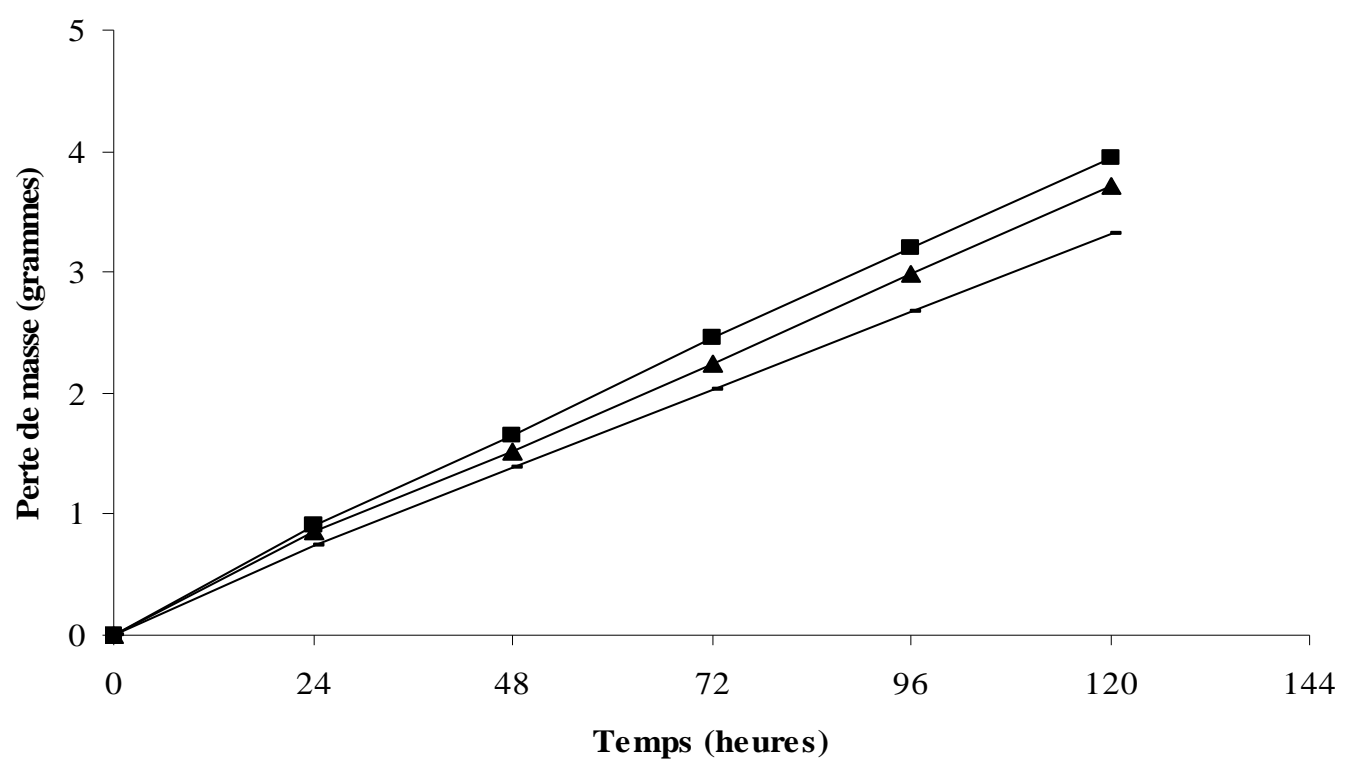

Figure 3: Profil de perméabilité à la vapeur d'eau (ロ film de pectine, $\boldsymbol{\Delta}$ film de gélatine, - film de gélatine/pectine). 


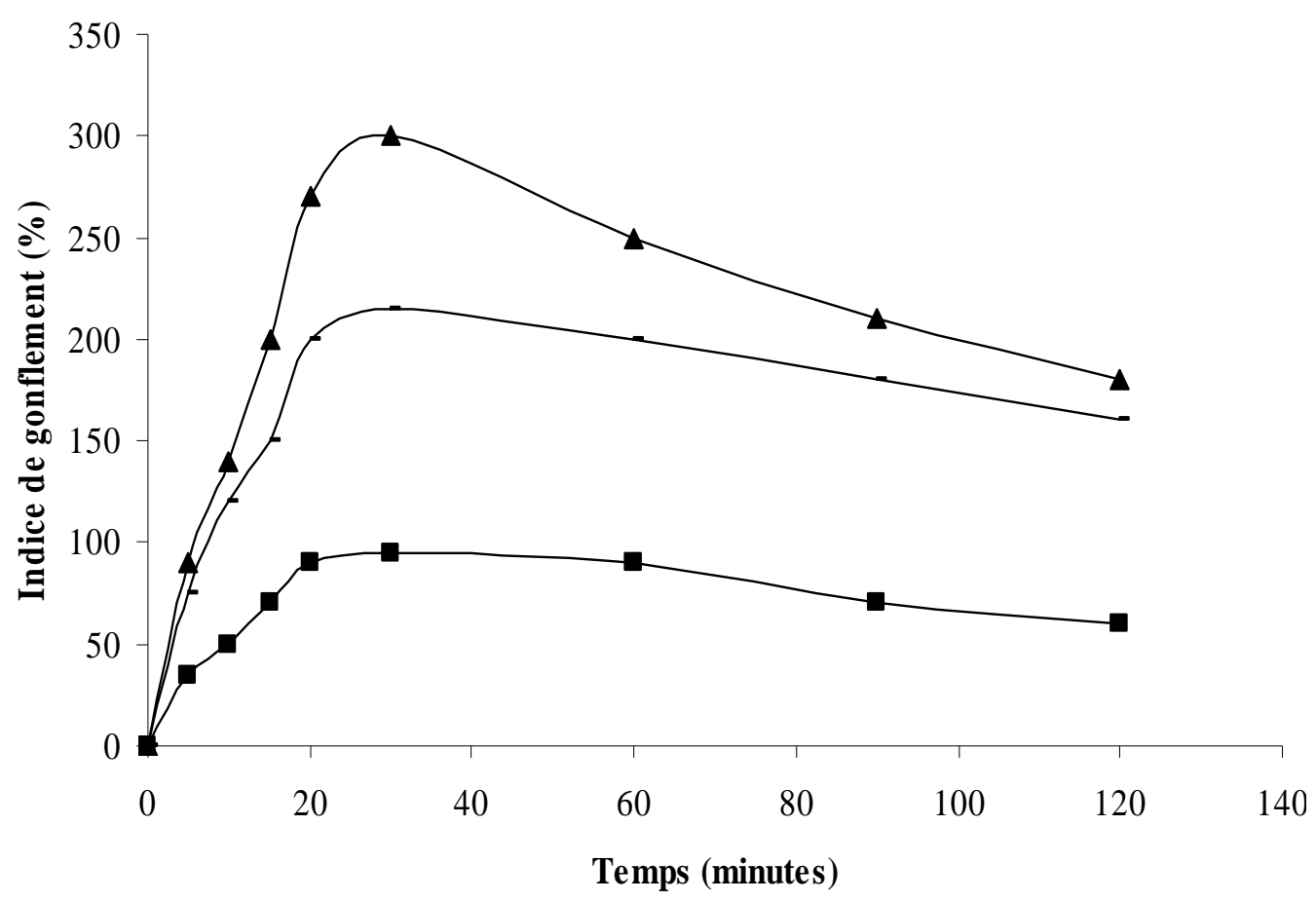

Figure 4: Indice de gonflement en fonction du temps ( $\boldsymbol{\Delta}$ film de gélatine, - film de gélatine/pectine, $\square$ film de pectine).

\section{Analyse morphologique}

Les résultats de la microscopie électronique à balayage (MEB) (figure 5) montrent que le film de gélatine (A) présente une surface homogène par endroit et serpentée à d'autres. Celui de la pectine (B) montre des agrégats de particules et une surface homogène par endroit. Quant au film du mélange gélatine/pectine $(\mathrm{C})$, il présente une surface homogène avec une zone fissurée avec quelques particules.

\section{DISCUSSION}

Selon Aulton (1982), les principales qualités d'un biofilm sont sa perméabilité, son pouvoir gonflant et son homogénéité. A concentration égale $(5 \%, \mathrm{~m} / \mathrm{v})$ et volume égal ( $5 \mathrm{ml}$ dispersés sur les boîtes de Pétri), le film de gélatine est apparu plus épais que celui de la pectine. Le mélange des deux polymères avait une épaisseur intermédiaire. Cette différence d'épaisseur a permis de différencier l'ensemble des films. Dans l'étude de Jo et al. (2005) ayant portée sur les mêmes polymères, une épaisseur de $0,17 \pm 0.01 \mu \mathrm{m}$ a été déclarée pour la pectine, la gélatine et le mélange gélatine/pectine sans aucune différence significative entre les films. Une telle valeur est très en dessous de celles mentionnées dans cette étude. Toutefois, cette différence pourrait s'expliquer par la concentration en polymères utilisée par les auteurs $(2,5 \% \mathrm{~m} / \mathrm{v})$, l'ajout de glycérol (agent plastifiant) et de polyvinylalcool (agent réticulant) dans la formulation, mais surtout par l'irradiation gamma qu'ont subi les films avant la mesure de leur épaisseur. En général, les films de faible épaisseur absorbent moins que les films épais (Fletcher et al., 1997). Un autre paramètre tout aussi important est le pouvoir gonflant des films lié à leur capacité d'absorption. Le pouvoir gonflant matérialisé par l'indice de gonflement est une propriété très étudiée au niveau des films pour applications alimentaires ou pharmaceutiques. Couplé à la perméabilité à la vapeur d'eau, ces deux paramètres évaluent la stabilité du film en conditions aqueuses. Comme l'ont déclaré Lee et al. (2004), un film utilisé pour emballer des aliments congelés ou des fruits frais découpés devra être moins perméable et posséder un indice de gonflement faible pour inhiber l'exsudation de ces aliments. Dans 

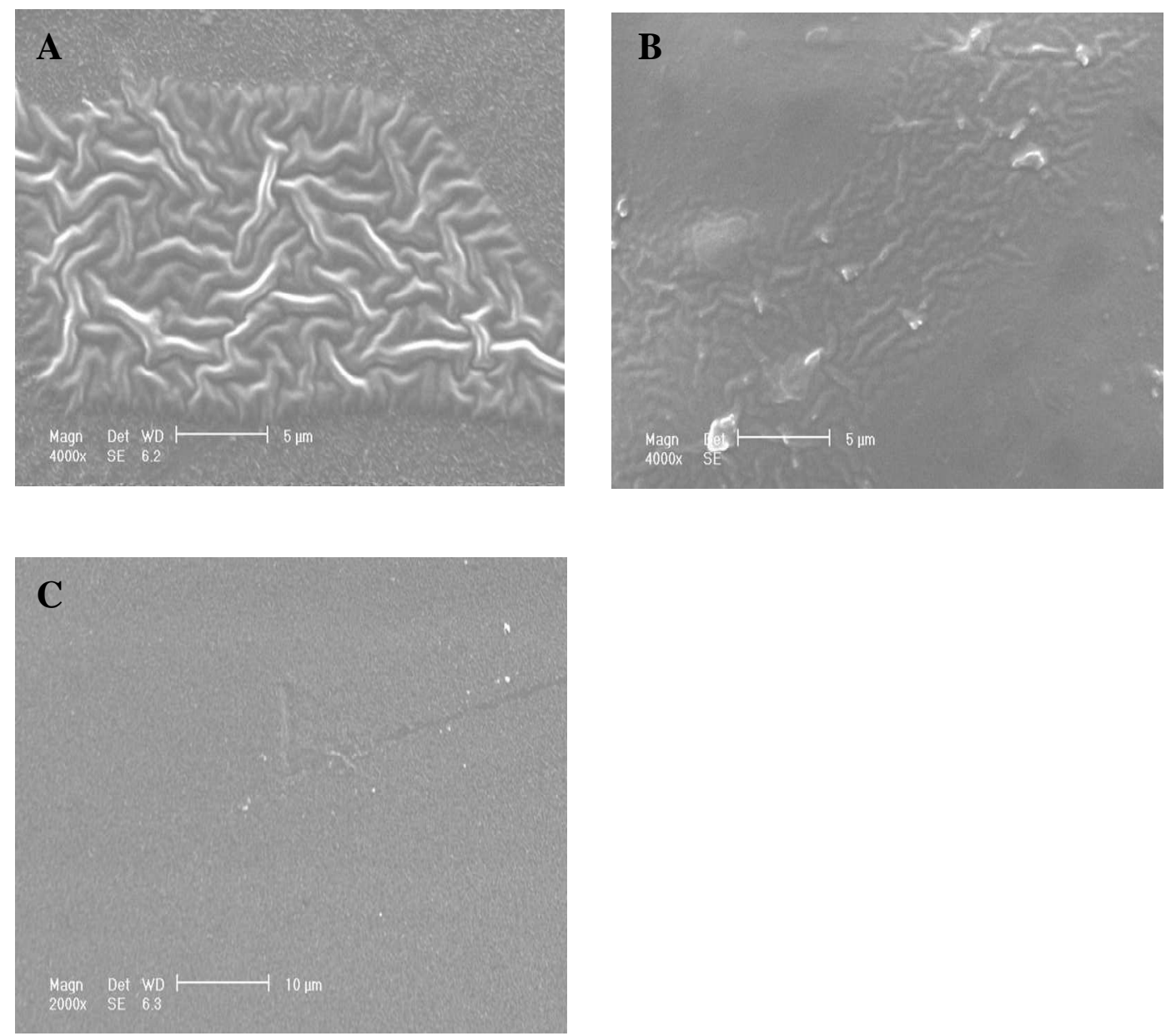

Figure 5: MEB de la surface des films (A: gélatine B: pectine C: gélatine/pectine).

cette étude, la pectine pourrait être le candidat idéal compte tenu de son indice de gonflement et de sa perméabilité les plus faibles. L'indice de gonflement de la pectine demeure en dessous de $100 \%$. Un indice de gonflement faible indique une tolérance élevée à l'eau. L'étude de l'indice de gonflement est un préalable pour une application efficiente d'un film de polymère. Le mélange pectine/gélatine possède un indice de gonflement de $215 \%$. Dans l'étude de Ghaffari et al. (2007) portant sur le mélange pectine/chitosan, un indice de gonflement d'environ $90 \%$ en milieu tampon phosphate $\left(\mathrm{pH}\right.$ 6) a été atteint dès la $30^{\mathrm{è}}$ minute. Cette valeur a été portée à environ $180 \%$ lorsque le $\mathrm{pH}$ était de 7,4 pour la même durée (30 min). Bien qu'il n'y ait pas de normes concernant les indices de gonflement des films issus de polymères naturels, les valeurs oscillent en général entre 0 et $400 \%$
(Dong et al., 2006; Ghaffari et al., 2007; Tapia et al., 2007). En revanche, un indice de gonflement élevé au-delà de $100 \%$ peut avoir des applications dans le domaine des pansements pour plaies et blessures superficielles ou chroniques (Reddy et al., 2006). Dawson et al. (1992) dans une étude clinique ont mis en évidence le pouvoir absorbant bénéfique des pansements à base d'alginate sur certaines infections cutanées. L'avantage résiderait dans l'absorption plus rapide de l'exsudation, ce qui faciliterait la cicatrisation de la blessure (Fletcher et al., 1997). Dans notre étude, la gélatine et le mélange gélatine/pectine ont respectivement affiché des indices de gonflement de $300 \%$ et $215 \%$. Les valeurs de perméabilité obtenues dans cette étude sont corrélées à celles de l'indice de gonflement. Le film de pectine était le moins perméable $\left(3,98 \mathrm{~g} \mathrm{~mm} / \mathrm{m}^{2} \mathrm{~h}\right.$ 
KPa) suivi du film du mélange $\left(6,59 \mathrm{~g} \mathrm{~mm} / \mathrm{m}^{2}\right.$ $\mathrm{h} \mathrm{KPa})$ et de celui de la gélatine $(9,20 \mathrm{~g}$ $\mathrm{mm} / \mathrm{m}^{2} \mathrm{~h} \mathrm{KPa}$ ). Cette perméabilité qui est une qualité essentielle des films est mise à profit dans la libération de substances actives (Dong et al., 2006; Silva et al., 2008; Yue et al., 2009). Dans l'étude de Jo et al. (2005) la perméabilité du mélange gélatine/pectine était de $4,86 \mathrm{~g} \mathrm{~mm} / \mathrm{m}^{2} \mathrm{~h} \mathrm{KPa}$ après $24 \mathrm{~h}$. Le film de gélatine obtenu dans la présente étude pourrait trouver des applications dans la libération accélérée de substances actives alors que celui de la pectine dans la libération prolongée ou retardée. En formant des films hybrides (mélange de polymères), on pourrait moduler la libération des substances actives.

Au niveau morphologique, les images de microscopie électronique montrent clairement des différences. Le film de gélatine présente une surface serpentée et homogène par endroit. Dans l'étude de Pranotoa et al. (2007) sur la gélatine de poisson, la microscopie électronique montre la structure interne du film formée de zone discontinue avec des fissures horizontales distribuées de manière aléatoire dans le réseau. Ces zones discontinues se formeraient lors du séchage de la solution filmogène (Bigi et al., 1998). Dans l'étude de Caoa et al. (2007) sur la gélatine type B issue de l'os de bovin, les auteurs montrent par microscopie électronique une structure interne lisse. Par contre, quand cette gélatine est mélangée à du tannin, on observe la présence d'une zone discontinue avec un aspect de fibrilles. Les auteurs attribueraient cette modification au processus de séchage ou à l'agent réticulant incorporé dans la formulation. Les images décrites dans ces études précédentes sont différentes de la nôtre. Gomez-Guillen et al. (2002) en étudiant plusieurs préparations de gélatine ont montré que le nombre de résidus d'acides aminés variait d'une préparation à une autre. La gélatine étant composée d'acides aminés, cette variabilité structurale pourrait être à l'origine des différences morphologiques observées dans notre étude et dans celles de Pranotoa et al. (2007) et Caoa et al. (2007). Le film de pectine quant à lui présente des agrégats de particules, cela pourrait s'expliquer par la nature même du composé qui est partiellement hydrosoluble, d'où la présence d'agrégats par endroit. L'absence d'image de microscopie électronique de la pectine à l'état natif ne nous permet pas de faire une comparaison objective avec notre étude. La littérature scientifique montre des images microscopiques de la pectine combinée à d'autres polymères comme le chitosan (Ghaffari et al., 2007) ou l'alginate (Da silva et al., 2009). L'une des rares études dans laquelle est présentée le mélange pectine/gélatine demeure celle de Jo et al. (2005) où les auteurs ont décrit une surface homogène du film. Selon Liu et al. (2007) l'inclusion de la gélatine au sein du mélange améliorerait les interactions moléculaires. Les auteurs ont décrit une structure homogène relativement bien organisée des films issus des mélanges gélatine/pectine et protéines de soja/pectine.

La structure homogène des films composés révélée dans les études précédentes et dans la nôtre apparaît être une caractéristique essentielle pour de nombreuses applications. Cette homogénéité pourrait être renforcée en modulant certains paramètres comme la concentration et le niveau de proportions des polymères. Cela pourra faire l'objet de travaux complémentaires de même que la possibilité d'incorporer des substances actives dans ces films et mesurer leur libération à partir de la matrice. Dans le futur, on pourrait envisager une meilleure compréhension des phénomènes physicochimiques se déroulant au sein de ces polymères lors de leur association. De nombreuses techniques sont décrites dans la littérature (spectroscopie infra rouge, résonance magnétique nucléaire, microcalorimétrie, thermogravimétrie, diffusion dynamique de la lumière etc). Malgré la complexité de ces techniques et les difficultés d'interprétation des résultats, des voies de recherches demeurent ouvertes.

\section{REFERENCES}

Akhgari A, Farahmand F, Garekani HA, Sadhegi F, Vandamme TF. 2006. Permeability and swelling studies on free films containing inulin in combination with different polymethacrylates aimed for colonic drug delivery. Eur. J. Pharm. Sci., 28: 307-314.

Arwidsson H. 1991. Properties of ethylcellulose films for extended release: influence of process factors when using 
organic solutions. Acta Pharm. Nord, 3: 25-30.

Aulton ME. 1982. Assessment of the mechanical properties of film coating materials. Int. J. Pharm. Tech. Prod. Manuf., 3: 9-16.

Avena-Bustillos RJ, Krochta JM, Salveit ME. 1997. Water resistance of red delicious apples and celery sticks coated with edible caseinate-acetylated monoglyceride films. J. Food Sci., 62(2): 351-354.

Bigi A, Bracci B, Gojazzi G, Panzavolta S, Roveri N. 1998. Drawn gelatin films with improved mechanical properties. Biomaterials, 19(24): 2335-2340.

Caoa N, Fua Y, Hea J. 2007. Mechanical properties of gelatin films cross-linked respectively by ferulic acid and tannin acid. Food Hydrocolloids, 21: 575-584.

Da Silva MA, Bierhalz ACK, Kieckbush TG. 2009. Alginate and pectin composite films crosslinked with calcium ions: effect of the plasticizer concentration. Carbohydrate Polymers, 77: 736-742.

Dawson C, Armstrong MWJ, Fulford SCV, Fauqi RM, Galland RB. 1992. Use of calcium alginate to pack abcess cavities. J. Royal Coll. Surg., 37: 177-179.

Dea ICM, Madden JK. 1986. Acetylated pectic polysaccharides of sugar beet. Food Hydrocolloids, 1: 71-88.

Dong Z, Wang Q, Du Y. 2006. Alginate/gelatin blend films and their properties for drug controlled release. $J$. Membrane Sci., 280: 37-44.

Dutta PK, Tripathi S, Mehrotra GK, Dutta J. 2009. Perspectives for chitosan based antimicrobial films in food applications. Food Chem., 114: 1173-1182.

Fletcher A, Cullum D, Trevor A, Sheldon THE. 1997. A systematic review of compression treatment for venous leg ulcers. Br. Med. J., 315: 576-580.

Fukunaka Y, Iwanaga K, Morimoto K, Kakemi M, Tabata Y. 2002. Controlled release of plasmid DNA from cationized gelatin hydrogels based on hydrogel degradation, J. Control. Release, 80(2): 333-343.

Garcia MA, Pinotti A, Martino MN, Zaritzky NE. 2004. Characterization of composite hydrocolloid films. Carbohydrate Polymers, 56: 39-345.
Ghaffari A, Navaee K, Oskoui M, Bayati KM, Rafiee-Tehrani. 2007. Preparation and characterization of free mixed-film of pectin/chitosan/Eudragit-RS intended for sigmoidal drug delivery. Eur. J. Pharm. Biopharm., 67: 175-186.

Gomez-Guillen MC, Turney J, FernandezDiaz MD, Ulmo N, Lizarbe MA, Montero P. 2002. Structural and physical properties of gelatin extracted from different marine species: a comparative study. Food Hydrocolloids, 16: 25-34.

Jo C, Kang H, Lee NY, Kwon JH, Byun MW. 2005. Pectin and gelatin-based films: effect of gamma irradiation on the mechanical properties and biodegradation. Rad. Phys. Che., 72: 745750.

Kozlov PV, Burdygina GI. 1983. The structure and properties of solid gelatin and the principles of their modification. Polymer, 24(6): 651-666.

Lee JY, Shim J, Lee HG. 2004. Mechanical properties of gellan and gelatin composite films. Carbohydrate Polymers, 56: 251254.

Liu L, Liu CK, Fishman ML, Hicks KB. 2007. Biodegradable composites from sugar beet pulp and poly(lactic acid). J. Agric. Food Chem., 55(6): 2349-2355.

Lofgren C, Hermansson AM. 2007. Synergistic rheological behavior of mixed HM/LM pectin gels. Food Hydrocolloids, 21(3): 480-486.

Lutz R, Aserin A, Wicker L, Garti N. 2009. Structure and physical properties of pectins with block-wise distribution of carboxylic acid groups. Food Hydrocolloids, 23: 786-794.

Neumann PM, Zur B, Ehrenreich Y. 1981. Gelatin-based sprayable foam as skin substitute. J. Biomater. Res., 15(1): 9-18.

Pranotoa Y, Leeb CM, Park HJ. 2007. Characterizations of fish gelatin films added with gellan and k-carrageenan. LWT, 40: 766-774.

Reddy M, Gill SS, Rochon PA. 2006. Prévention des escarres: une revue systématique. J. Amer. Med. Ass., 296(8): 974-984.

Ross-Murphy SB. 1992. Structure and rheology of gelatin gels: recent progress. Polymer, 33(12): 2622-2627. 
Samuelsen AB, Paulsen BS, Wold JK, Otsuka $\mathrm{H}$, Kiyohara $\mathrm{H}$, Yamadab $\mathrm{H}$, Knutsenc SH. 1996. Characterization of biologically active pectin from Plantago major L. Carbohydrate Polymers, 30: 3744.

Silva CL, Pereira JC, Ramalho A, Pais AACC, Sousa JJS. 2008. Films based on chitosan polyelectrolyte complexes for skin drug delivery: Development and characterization. J. Membrane Sci., 320: 268-279.

Tabata Y, Ikada Y. 1998. Protein release from gelatin matrices. Adv. Drug Deliv. Rev., 31(2): 287-301.

Tapia MS, Rojas-Grau MA, Rodriguez FJ, Ramirez J, Carmona A, Mertin-Belloso O. 2007. Alginate and gellan-based edible films for probiotics coatings or fresh cut fruits. J. Food Sci., 72(4): 190-196.

Yue Y, Sheng X, Wang P. 2009. Fabrication and characterization of microstructured and $\mathrm{pH}$ sensitive interpenetrating networks hydrogel films and application in drug delivery field. Eur. Polymer J., 45: $309-315$.

Zactiti EM, Kieckbusch TG. 2006. Potassium sorbate permeability in biodegradable alginate films: Effect of the antimicrobial agent concentration and crosslinking degree. J. Food Engin., 77: 462-467.

Zaleska H, Ring S, Tomasik P. 2001. Electrosynthesis of potato starch-whey protein isolate complexes. Carbohydrate Polymers, 45: 89-94. 